\title{
The Discrete Boltzmann Equation : The Regular Plane Model with Four Velocities
}

\author{
Henri Cabannes ${ }^{1}$ and Li-Shi Luo ${ }^{2}$ \\ ${ }^{1}$ Laboratoire de Modélisation en Mécanique, Université Paris VI, \\ 4 place Jussieu, 75005 Paris - France \\ Email : henri.cabannes@normalesup.org \\ ${ }^{2}$ National Institute of Aerospace, \\ 144 Research Drive, Hampton, VA 23666, USA \\ Email : $\underline{\text { luo@ nianet.org }}$
}

\begin{abstract}
For a simple discrete model of Boltzmann equation, we study the derivatives of $\mathrm{H}$ Boltzmann function, and prove that all derivatives of odd order are negative, instead all derivatives of even order are postive. These result is a first and small generalisation of the classical $H$-Boltzmann theorem.
\end{abstract}

We consider a discrete model of the Boltzmann equation with the the four following velocities : $\boldsymbol{\xi}_{1}=\boldsymbol{c}(1,0), \boldsymbol{\xi}_{2}=\boldsymbol{c}(0,1), \boldsymbol{\xi}_{3}=\boldsymbol{c}(-1,0), \boldsymbol{\xi}_{4}=\boldsymbol{c}(0,-1)$. The only binary collisions are $\left(\boldsymbol{\xi}_{1}, \boldsymbol{\xi}_{3}\right) \leftrightarrow\left(\boldsymbol{\xi}_{2}, \boldsymbol{\xi}_{4}\right)$. Denoting by $\boldsymbol{N}_{\boldsymbol{i}}(\boldsymbol{t})$ the density of molecules with velocity $\boldsymbol{\xi}_{\mathrm{i}}$, and by $\boldsymbol{H}(\boldsymbol{t})=\sum_{i=1}^{4} N_{i}(t) \log \left|N_{i}(t)\right|$, the $H$-Boltzmann functional, we prove the following result :

$$
(-1) \frac{k}{d t} \frac{d^{k} H(t)}{k} \geq 0
$$

This result was published by Harris in 1967, [1], but unfortunately the proof of Harris was erroneous, as he has recognized in two e-mails. In the first e-mail, sent to Li-Shi Luo (on October 13, 2003) he writes : "I am impressed how elegant your proof is, ... . It is a truly wonderful piece of work and I am glad to see the matter resolved after so many years of thinking about it ". In the second e-mail, sent to Henri Cabannes (on October 20, 2003) he writes : " Congratulations on your proof which I am in great admiration of ..... . I would be interested to see for which, if any, other models a proof can be found ". Harris passed away last May 2004, at the age of 67 ! We have given an exact proof, last novembre, in reference [2], which is the section 3.1.3 of Lecture Notes on The Discrete Boltzmann Equation.

\subsubsection{The $\boldsymbol{H}$-Boltzmann Function}

The first derivative of the $H$-Boltzmann function is negative. It is interesting to note that for the regular plane four velocities model, it is true that the successive derivatives of the $H$-Boltzmann function alternate in sign [45]:

$$
(-1)^{k} \frac{d^{k} H}{d t^{k}} \geq 0, \quad k=1,2, \ldots
$$

CP762, Rarefied Gas Dynamics: $24^{\text {th }}$ International Symposium, edited by M. Capitelli (C) 2005 American Institute of Physics 0-7354-0247-7/05/\$22.50 
As a consequence of the first Euler equation, when the densities are independent of the space variables, the total density $n$ is a constant. Letting $n_{i}=N_{i} / n$ and $\tau=c S n t$, we can write the kinetic equations (3.1.1-1) as:

$$
\frac{d n_{i}}{d \tau}=n_{i+1} n_{i+3}-n_{i} n_{i+2}, \quad i=1,2,3,4, \quad \text { with }\left(n_{1}+n_{2}+n_{3}+n_{4}\right)=1
$$

In the above equation we are considering $n_{k}=n_{l}$ when $k \equiv l(\bmod 4)$. From equations (3.1.3-2) we deduce:

$$
\frac{d^{k} n_{i}}{d \tau^{k}}=(-1)^{k+1} \frac{d n_{i}}{d \tau}, \quad i=1,2,3,4
$$

The $H$-Boltzmann function is:

$$
H=\sum_{i=1}^{4} N_{i} \ln \left(N_{i}\right)=n \ln (n)+n \sum_{i=1}^{4} n_{i} \ln \left(n_{i}\right),
$$

and because $n$ is a positive constant, the derivatives with respect to $t$ of $H$ have the same sign as the derivatives with respect to $\tau$ of:

$$
h(\tau)=\sum_{i=1}^{4} n_{i}(\tau) \ln \left(n_{i}(\tau)\right) .
$$

By taking successive derivatives we obtain:

$$
\begin{aligned}
\frac{d h}{d \tau} & =\sum_{i=1}^{4} \ln \left(n_{i}\right) \frac{d n_{i}}{d \tau}=\left(n_{1} n_{3}-n_{2} n_{4}\right) \ln \left(\frac{n_{2} n_{4}}{n_{1} n_{3}}\right) \leq 0, \\
\frac{d^{2} h}{d \tau^{2}} & =\sum_{i=1}^{4}\left\{\ln \left(n_{i}\right) \frac{d^{2} n_{i}}{d \tau^{2}}+\frac{1}{n_{i}}\left(\frac{d n_{i}}{d \tau}\right)^{2}\right\} \\
& =-\frac{d h}{d \tau}+\sum_{i=1}^{4} A_{i}, \quad A_{i}:=\frac{1}{n_{i}}\left(\frac{d n_{i}}{d \tau}\right)^{2} \\
\frac{d^{k+2} h}{d \tau^{k+2}} & =-\frac{d^{k+1} h}{d \tau^{k+1}}+\frac{d^{k} A}{d \tau^{k}}, \quad \text { with } \quad A:=\sum_{i=1}^{4} A_{i} .
\end{aligned}
$$

The initial values of the densities $\left\{N_{i}\right\}$ are positive, and so is the initial value of $A$ and the derivative $\frac{d^{2} h}{d \tau^{2}}$.

To complete the proof of inequalities (3.1.3-1) it suffices to show that:

$$
(-1)^{k} \frac{d^{k} A_{i}}{d \tau^{k}} \geq 0 .
$$

This will certainly be true if we can show:

$$
(-1)^{k} \frac{d^{k} A_{i}}{d \tau^{k}} \geq A_{i} \quad \forall k,
$$

because $A_{i} \geq 0$. The above inequality can be proved by induction. 
For $k=1$ we have:

$$
-\frac{d A_{i}}{d \tau}=\frac{1}{n_{i}}\left\{2\left(\frac{d n_{i}}{d \tau}\right)^{2}+A_{i} \frac{d n_{i}}{d \tau}\right\}=A_{i}\left\{2+\frac{1}{n_{i}} \frac{d n_{i}}{d \tau}\right\} .
$$

Equation (3.1.3-2) can be written as:

$$
n_{i}+\frac{d n_{i}}{d \tau}=n_{i+1} n_{i+3}+n_{i}\left(n_{i-1}+n_{i}+n_{i+1}\right) \geq 0
$$

which proves inequality (3.1.3-6) for $k=1$. To compute $\frac{d^{k} A_{i}}{d \tau^{k}}$, we differentiate the product $n_{i} A_{i}$ in two different ways. First we use formula (3.1.3-3) and then we use Leibniz rule:

$$
\begin{aligned}
\frac{d^{k}\left(n_{i} A_{i}\right)}{d \tau^{k}} & =\frac{d^{k}}{d \tau^{k}}\left(\frac{d n_{i}}{d \tau}\right)^{2}=(-2)^{k}\left(\frac{d n_{i}}{d \tau}\right)^{2} \\
\frac{d^{k}\left(n_{i} A_{i}\right)}{d \tau^{k}} & =\sum_{j=0}^{k-1} C_{k}^{j} \frac{d^{k-j} n_{i}}{d \tau^{k-j}} \frac{d^{j} A_{i}}{d \tau^{j}}+n_{i} \frac{d^{k} A_{i}}{d \tau^{k}}
\end{aligned}
$$

where $C_{k}^{j}:=k ! / j !(k-j) !$ is the binomial coefficient. Comparing the last two equations yields:

$$
(-1)^{k} \frac{d^{k} A_{i}}{d \tau^{k}}=\frac{1}{n_{i}}\left\{2^{k}\left(\frac{d n_{i}}{d \tau}\right)^{2}+\sum_{j=0}^{k-1}(-1)^{j} C_{k}^{j} \frac{d^{j} A_{i}}{d \tau^{j}} \frac{d n_{i}}{d \tau}\right\} .
$$

We have shown inequality (3.1.3-6) holds for $k=1$, assume that it holds for $(k-1)$, then the above equality leads to:

$$
\begin{aligned}
(-1)^{k} \frac{d^{k} A_{i}}{d \tau^{k}} & \geq A_{i}\left\{2^{k}+\frac{1}{n_{i}} \frac{d n_{i}}{d \tau} \sum_{j=0}^{k-1} C_{k}^{j}\right\} \\
& =A_{i}\left\{2^{k}+\left(2^{k}-1\right) \frac{1}{n_{i}} \frac{d n_{i}}{d \tau}\right\} \\
& =A_{i}\left\{1+\left(2^{k}-1\right) \frac{1}{n_{i}}\left(n_{i}+\frac{d n_{i}}{d \tau}\right)\right\} \\
& \geq A_{i} .
\end{aligned}
$$

This completes the proof of inequality (3.1.3-6), and hence forth inequality (3.1.3-1).

The densities $\left\{N_{i}(t)\right\}$ are monotonic functions of time, and if the initial state is Maxwellian so that $\left(\bar{n}_{1} \bar{n}_{3}-\bar{n}_{2} \bar{n}_{4}\right)=0$, then the $\left\{N_{i}(t)\right\}$ are constants.

\section{Conclusion.}

To conclude our work and to answer the second Harris's e-mail, we can suggest different possible extensions. First : one can try to extend our results to the three dimensional Broadwell model, with six velocities, or more generally models with $2 p$ velocities ; one can try to prove that the second derivative of $\boldsymbol{H}$ Boltzmann functional is, at least for those 
models, always positive. The second suggestion is to study the same problem for the twodimensional semi-continuous model of Boltzmann equation

$$
\frac{\partial N(t ; \theta)}{\partial t}=\frac{1}{2 \pi} \int_{0}^{2 \pi}\{N(t ; \phi) N(t ; \phi+\pi)-N(t ; \theta) N(t ; \theta+\pi)\} d \phi
$$

All the velocities have the same modulus, and arbitrary directions. The unknown function $\boldsymbol{N}(\boldsymbol{t} ; \boldsymbol{\theta})$, a density, depends upon time $\boldsymbol{t}$ and and angle $\boldsymbol{\theta}$, direction of velocity. $\boldsymbol{N}(\boldsymbol{t} ; \boldsymbol{\theta})$ is a periodic function in $\boldsymbol{\theta}$, with period $2 \pi$; when the period is equal to $\pi$, the general solution was obtained in parametric form [3], [4], [5]. An interesting problem seems to be the determination of all initial densities $\boldsymbol{N}(0 ; \boldsymbol{\theta})$, for which the second derivative of $\boldsymbol{H}$ Boltzmann functional is positive. Our conjecture is that positivity is valid for all solutions.

\section{REFERENCES}

[1] Harris Stewart - Proof that Successive Derivatives of Boltzmann's H Function for a Discrete Velocity Gas Alternate in Sign. Journal of Mathematical Physics, 8, pp. 2407-2410, (1967).

[2] Cabannes Henri, Gatignol Renée \& Luo li-Shi - The Discrete Boltzmann Equation, electronic revised version, in http://lapasserelle.com/henri_cabannes, under Principales publications/Livres, pp. 27-29, (2003)

[3] Sibgatullin N. and Cabannes H. - Homogenous solutions for a semi-continuous model of the Boltzmann equation. Math. Models and Methods in Appied Sciences. 5, 1129-1138 (1995).

[4] Cabannes H. and Sibgatullin N. - Analytic solution for a semi-continuous model of the Boltzmann equation. Proceedings of $20^{\text {th }}$ International Symposium, Pekin Univ. Press, pp. 4348 , Beijing (1997).

[5] Cabannes H. and Sibgatullin N. - The Cauchy problem for a semi-continuous model of the two-dimensional homogeneous Boltzmann equation. C.R.Acad. Sci. Paris, 325 , pp. 217222, (1997). 